ARTICLE

\title{
Charge density waves and Fermi surface reconstruction in the clean overdoped cuprate superconductor $\mathrm{Tl}_{2} \mathrm{Ba}_{2} \mathrm{CuO}_{6+\delta}$
}

C. C. Tam (1) ${ }^{1,2}$, M. Zhu' ${ }^{1}$, J. Ayres (10 ${ }^{1}$, K. Kummer (1) ${ }^{3}$, F. Yakhou-Harris ${ }^{3}$, J. R. Cooper ${ }^{4}$, A. Carrington (i) ${ }^{1 凶} \&$ S. M. Hayden (1) ${ }^{1 \times}$

Hall effect and quantum oscillation measurements on high temperature cuprate superconductors show that underdoped compositions have small Fermi surface pockets whereas when heavily overdoped, a single much larger pocket is found. The origin of this change in electronic structure has been unclear, but may be related to the high temperature superconductivity. Here we show that the clean overdoped single-layer cuprate $\mathrm{Tl}_{2} \mathrm{Ba}_{2} \mathrm{CuO}_{6+\delta}$ (TI2201) displays CDW order with a remarkably long correlation length $\xi \approx 200 \AA$ which disappears above a hole doping of $p_{\mathrm{CDW}} \approx 0.265$. We show that the evolution of the electronic properties of TI2201 as the doping is lowered may be explained by a Fermi surface reconstruction which accompanies the emergence of the CDW below $p_{\mathrm{CDW}}$. Our results demonstrate importance of CDW correlations in understanding the electronic properties of overdoped cuprates.

\footnotetext{
${ }^{1} \mathrm{H}$. H. Wills Physics Laboratory, University of Bristol, Bristol BS8 1TL, United Kingdom. ${ }^{2}$ Diamond Light Source, Harwell Campus, Didcot OX11 ODE, United Kingdom. ${ }^{3}$ ESRF, The European Synchrotron, 71 Avenue des Martyrs, CS40220, 38043 Grenoble Cedex 9, France. ${ }^{4}$ Department of Physics, University of Cambridge, Madingley Road, Cambridge CB3 OHE, United Kingdom. ${ }^{凶}$ email: a.carrington@bristol.ac.uk; s.hayden@bristol.ac.uk
} 
T he normal state electronic properties of the hole doped cuprate superconductors evolve dramatically with doping. This evolution, in part, evidences the emergence and disappearance of competing ordering tendencies and their fluctuations, namely those associated with the pseudogap, charge and $\operatorname{spin}^{1}$. Fluctuations associated with these orders are enhanced close to their putative quantum critical points and have been conjectured to play an important role in producing the high superconducting transition temperature in these materials.

The pseudogap corresponds to a loss of electronic states near the Fermi energy ${ }^{2,3}$ and in most, if not all, cuprates occurs for doping $p<p^{\star} \approx 0.19^{3,4}$. In this regime a charge density wave (CDW) order is also found ${ }^{5-11}$. In $\mathrm{YBa}_{2} \mathrm{Cu}_{3} \mathrm{O}_{6+x}$ (YBCO), charge order ${ }^{5,6}$ is the obvious candidate responsible for the reconstruction of the Fermi surface $^{12,13}$ resulting in closed pockets observed by quantum oscillations ${ }^{14}$. The onset of the CDW ${ }^{5,6}$ also naturally explains ${ }^{12}$ a sign reversal observed ${ }^{15}$ in the Hall number $n_{H}$ at low-temperature for doping $p<0.16$. At slightly higher doping, $n_{H}$ in the high-field, low-temperature limit $n_{H}^{\infty}$ undergoes a rapid increase from $p \rightarrow 1+p$ over a narrow doping range $0.16<p<0.20$, which has been suggested to be caused by the closing of the pseudogap ${ }^{16,17}$. In the overdoped cuprate $\mathrm{Tl}_{2} \mathrm{Ba}_{2} \mathrm{CuO}_{6+\delta}$ (Tl2201), a similar $p \rightarrow 1+p$ transition is observed in $n_{H}^{\infty}$ which onsets at a higher doping $p \simeq 0.25$, where thermodynamic probes suggest that there is no pseudogap ${ }^{18-21}$. Furthermore as, prior to the present work, there were no reports of a CDW in overdoped Tl2201, a universal microscopic origin of the transition in $n_{H}^{\infty}$ remains to be found. Here we use $\mathrm{Cu}-L_{3}$ edge resonant inelastic x-ray scattering (RIXS) to observe a CDW in heavily overdoped T12201. The doping onset of the CDW coincides with the decrease in $n_{H}^{\infty}$ in Tl2201 suggesting these phenomena are linked.

The single-layer cuprate, Tl2201, is exceptionally electronically clean and is the only hole doped cuprate in which the large $(1+p)$ Fermi surface has been observed by quantum oscillations $(\mathrm{QO})^{22-24}$. The shape of the Fermi surface for the most overdoped compositions $(p \geq 0.27)$ has been determined by angle-dependent magnetoresistance 25,26 , QO and angle resolved photoemission spectroscopy (ARPES) ${ }^{27}$ and is in excellent agreement with conventional density functional theory ${ }^{24}$. Previously, there have been no reports of charge or other ordering in this overdoped material. However CDWs have been seen in other overdoped cuprates. In $\mathrm{La}_{2-x} \mathrm{Sr}_{x} \mathrm{CuO}_{4}$ (LSCO), the CDW has been seen to persist into the overdoped regime (up to $p=0.21)^{28}$, and a second CDW phase, disconnected from the $\mathrm{CDW}$ at lower doping, was observed in overdoped $(\mathrm{Bi}, \mathrm{Pb})_{2.12} \mathrm{Sr}_{1.88} \mathrm{CuO}_{6+\delta} \quad(\mathrm{Bi} 2201)^{29}$. In overdoped $\mathrm{Bi}_{2} \mathrm{Sr}_{2} \mathrm{CaCu}_{2} \mathrm{O}_{8+\delta}(\mathrm{Bi} 2212)$ no $\mathrm{CDW}$ is seen directly ${ }^{30}$ although there is phonon softening which may be a precursor of CDW formation.

\section{Results}

Charge density wave order in $\mathrm{Tl}_{2} \mathrm{Ba}_{2} \mathrm{CuO}_{6+\delta}$. Charge density wave correlations in underdoped superconducting cuprates ${ }^{5-11}$ have ordering wavevectors with in-plane components along the $\mathrm{CuO}$ bonds i.e. $(\delta, 0)$ and $(0, \delta)$, where $0.23 \lesssim \delta \lesssim 0.33$ r.l.u. In the absence of a large magnetic field or uniaxial stress, a 2D CDW develops below $T_{\mathrm{CDW}}$. This state corresponds to a weak anticorrelation of the phase of the $\mathrm{CDW}$ in neighbouring $\mathrm{CuO}_{2}$ planes and produces a peak in the scattered intensity at halfinteger $L$ positions such as $(\delta, 0,2.5)$. In this paper, we label reciprocal space in reciprocal lattice units (r.l.u.) where $\mathbf{Q}=$ $H \mathbf{a}^{\star}+K \mathbf{b}^{\star}+L \mathbf{c}^{\star}$. We use RIXS (see Methods) to study the charge correlations in three overdoped superconducting samples. Our samples have critical temperatures $T_{\mathrm{c}}=56,45$ and $22 \mathrm{~K}$, corresponding to hole doping $p=0.23, p=0.25$ and $p=0.28^{21}$. Throughout the paper we probe CDW correlations with $L=2.5$, which allows us to scan a wide range of $H$ (see Supplementary Note 1, Supplementary Fig. 1 and Supplementary Table 1).

Figure la shows a typical RIXS energy $(E)$ dependent spectrum obtained in this experiment showing $d d$ excitations, corresponding to a transition between $2 p \rightarrow 3 d$ states, near $E=2 \mathrm{eV}$ and features at lower energy which may be fitted (see Methods) with an elastic peak, a non-elastic peak near $60 \mathrm{meV}$ and a broad peak centred at about $150 \mathrm{meV}$. We believe the broad $150 \mathrm{meV}$ peak is due to paramagnon spin fluctuations previously observed in $\mathrm{Tl} 2201^{31}$ and the $60 \mathrm{meV}$ peak is due to a phonon.

Figure 2 shows data such as that in Fig. 1 for the $p=0.25$ $\left(T_{c}=45 \mathrm{~K}\right)$ sample compiled into $H-E$ colour maps. The spectra can be fitted to the three components mentioned above. The elastic intensity, obtained by integrating the RIXS intensity between $-100<E<100 \mathrm{meV}$ (Fig. 2c) is peaked at $H \approx \pm 0.31$. These elastic features are also seen in Fig. $2 \mathrm{a}$, and we interpret them as being due to charge density wave order as explained below. The elastic scattering is also peaked at $H=0$ due to specular scattering from

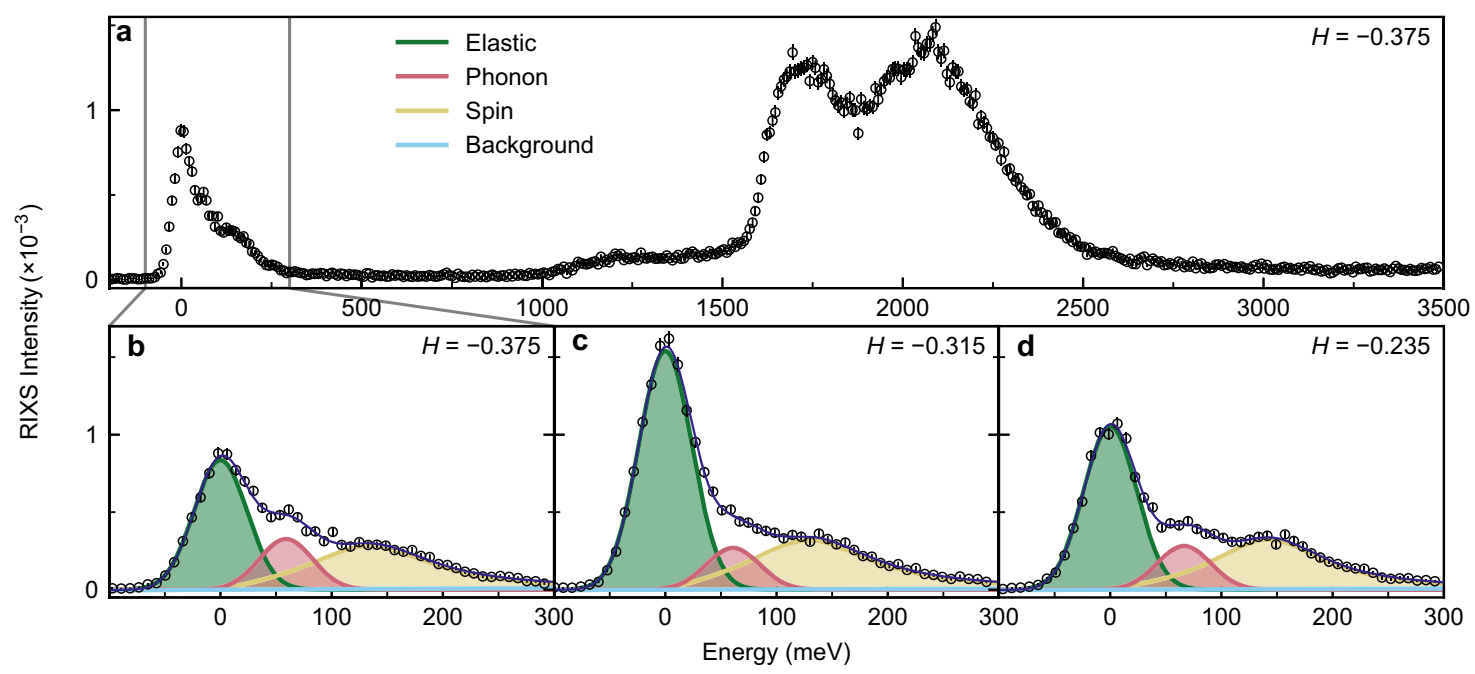

Fig. 1 RIXS spectra. a RIXS spectra of the $p=0.25$ sample taken at $45 \mathrm{~K}$ showing $d d$ excitations near 2000 meV. b-d The low-energy region of the RIXS spectra, can be fitted with three components comprising elastic scattering including the CDW, phonon and paramagnon spin excitations (see Methods). The elastic scattering is strongest at $H=-0.315$ where the CDW is present. We label reciprocal space using $\mathbf{Q}=H \mathbf{a}^{\star}+K \mathbf{b}^{\star}+L \mathbf{c}^{\star}$, where $\left|\mathbf{a}^{\star}\right|=2 \pi / a$, $a=b=3.85 \AA, c=23.1 \AA$, and negative $H$ implies grazing incident $x$-rays 

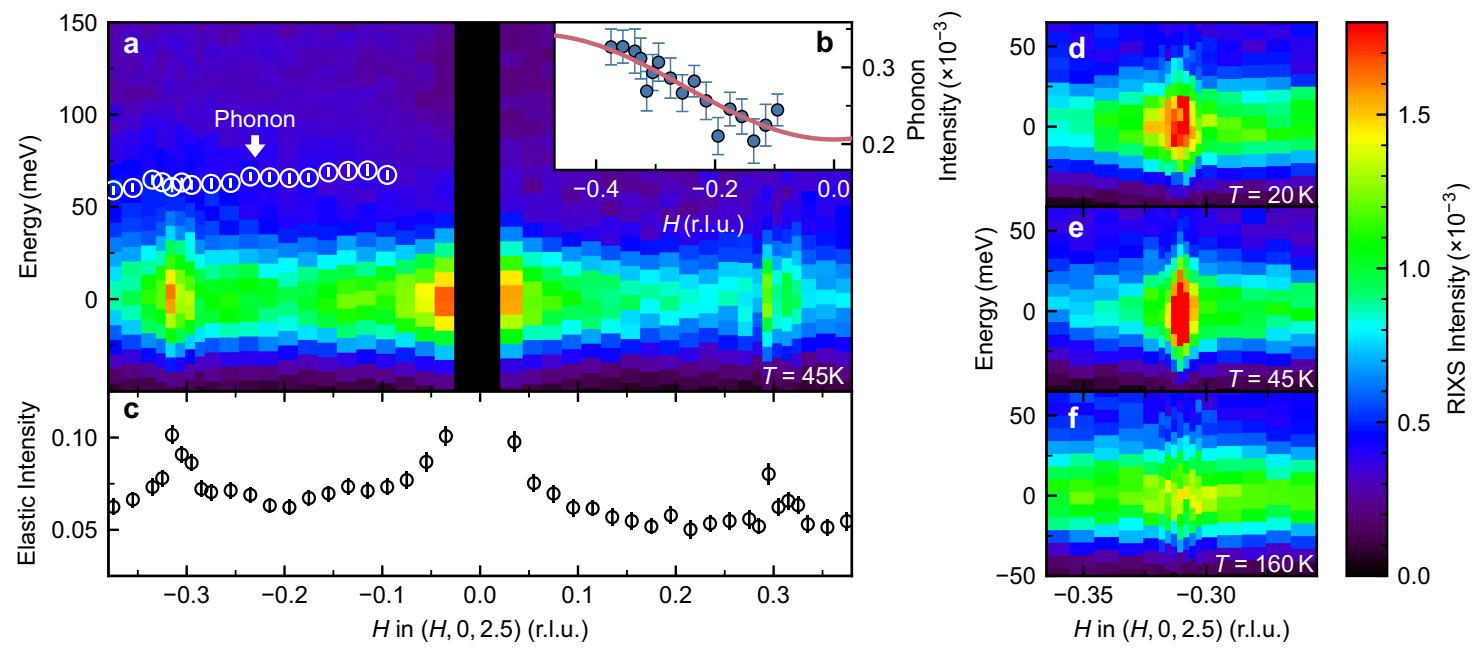

Fig. 2 RIXS intensity maps. Maps for $p=0.25$ sample taken at $45 \mathrm{~K}$. a $H-E$ map over the $H$ range \pm 0.375 . White circles are the energies of the feature in Fig. $1 \mathbf{b}$, $\mathbf{c}$ believed to be a phonon and the intensity of the feature is plotted in $\mathbf{b}$, along with a line proportional to $\sin ^{2}(\pi H)$ in red. $\mathbf{c}$ The elastic intensity obtained by integrating the RIXS intensity between $-100<E<100 \mathrm{meV}$, and dividing by the integral between $1000<E<3000$ meV. Peaks at $H \approx \pm 0.31$ are due to the charge density wave. $\mathbf{d}-\mathbf{f}$ are RIXS intensity maps of the peak centred at $H \approx-0.31$ at different temperatures.

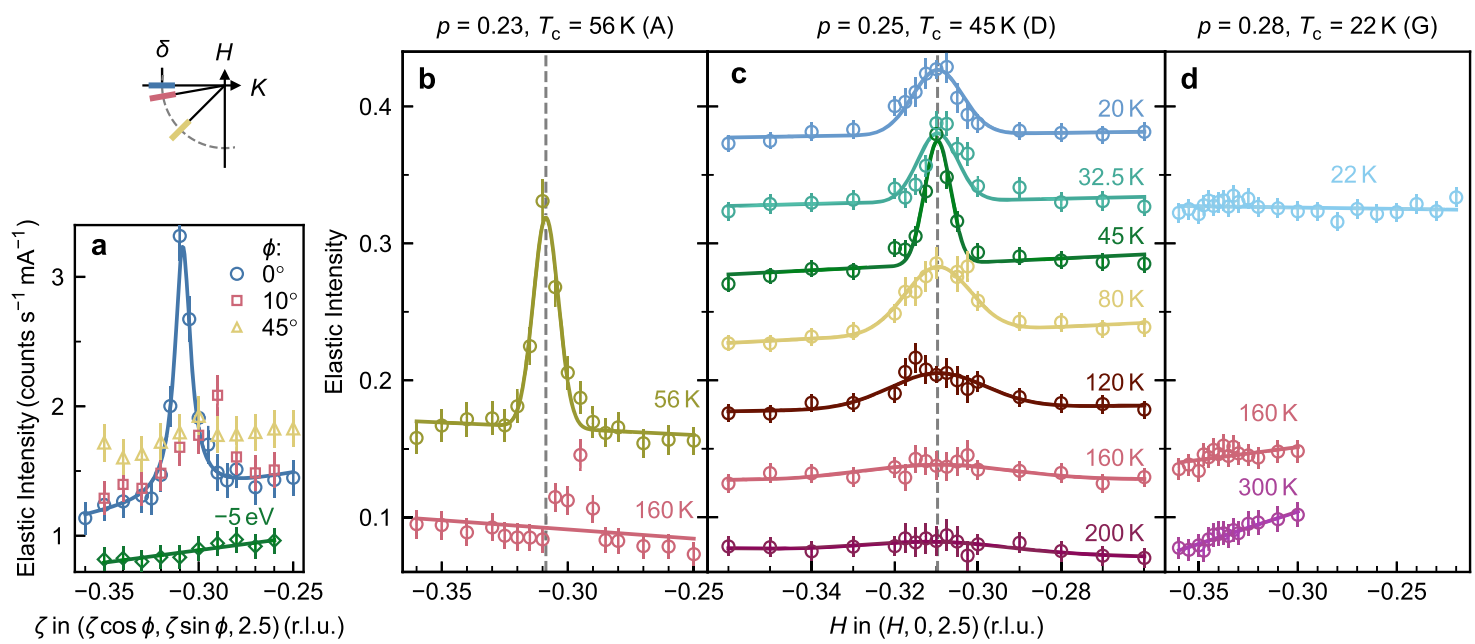

Fig. 3 Wavevector-dependent scans of the CDW order. Elastic intensities, obtained integrating RIXS intensity over energy in the range $-100<E<100 \mathrm{meV}$, of three samples of T12201 at various dopings. a Scans along lines $(\zeta \cos \phi, \zeta \sin \phi, 2.5)$ showing CDW order occurs near $(-0.31,0$, 2.5) and off-resonance scan with $\phi=0$ for $p=0.23$. Measurements are normalised to the storage ring current. The sketch above indicates the direction of the scans in the $(H, K)$ plane. $\mathbf{b}-\mathbf{d}(H, 0,2.5)$ scans, with intensities normalised to the $d d$ excitations. Measurements at successively lower temperatures in c have been offset by a value of 0.05 units per temperature. Data has been fitted to a Gaussian with standard deviation parameter $\sigma$ plus linear background. The grey dashed line marks the centre of the Gaussian peak at $T_{\mathrm{c}}$ which we have used to determine $\delta$.

disorder in the sample surface. Note that charge scattering is generally enhanced for grazing incident-geometry (denoted by negative $H$ ) and the vertical polarisation used here. The energy of the fitted phonon peak is plotted as white circles (Fig. 2a). Comparing the dispersion and the $\sin ^{2}(\pi H)$ intensity variation (Fig. 2b) to theory ${ }^{32}$ we conclude that we observe the $\mathrm{CuO}$ bondstretching mode previously seen in Cu- $L$ RIXS on cuprates $^{33}$. The $\sin ^{2}(\pi H)$ intensity variation can understood in terms of a momentum dependent electron-phonon coupling ${ }^{28,34}$. Phonon softening has been observed in other cuprates such as $\mathrm{La}_{2-x} \mathrm{Sr}_{x} \mathrm{CuO}_{4}{ }^{28}$ for $H>\delta$. Our data is consistent with a softening of up to $10 \%$, but our experiment is not able to say definitively whether there is phonon softening or not and further work is required (see Supplementary Note 2 and Supplementary Figs. 2, 3).

In order to further characterise this CDW feature we investigated other reciprocal space positions and performed non-resonant x-ray scattering. Figure 3a shows elastic intensity scans obtained by integrating the RIXS intensity over the energy range $-100<E<100$ $\mathrm{meV}$ for the sample with doping $p=0.23$ at a temperature $T=T_{c}=56 \mathrm{~K}$. The open circles show a scan along $(H, 0,2.5)$ yielding a peak at $H=-0.31$ r.l.u. This peak is not present when scans are made along $(\zeta \cos \phi, \zeta \sin \phi, 2.5)$, with $\phi=10^{\circ}$ or $45^{\circ}$, nor for non-resonant scattering when the incident photon energy was detuned from resonance by $5 \mathrm{eV}$. From this, we conclude that the data is consistent with a CDW with an in-plane propagation vector of $(\delta, 0)$ as observed in other cuprates. A high-energy $(500<E<900 \mathrm{meV})$ inelastic ring-like feature in $(H, K)$ was observed in $\mathrm{Bi}_{2} \mathrm{Sr}_{2} \mathrm{CaCu}_{2} \mathrm{O}_{8+\delta}$, which was interpreted as evidence for dynamical charge fluctuations ${ }^{35}$. We find no evidence for a feature like this in our $\zeta$ scans (see Supplementary Note 3 and Supplementary Fig. 4).

Temperature and doping dependence, correlation length. Figure $3 \mathrm{~b}-\mathrm{d}$, show $H$-scans through the expected position of the 


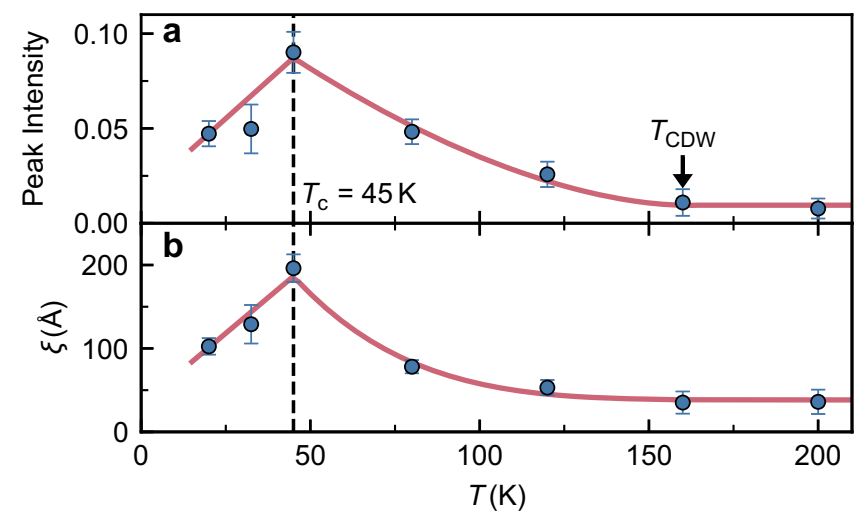

Fig. 4 Temperature dependence of CDW intensity and correlation length. The CDW peak intensity $\mathbf{a}$ and the $a$-axis correlation length $\mathbf{b}$ of the $p=0.25$ sample. The dashed line marks $T_{\mathrm{c}}=45 \mathrm{~K}$ and the arrow marks $T_{\mathrm{CDW}}=160 \mathrm{~K} \cdot \xi\left(T=T_{\mathrm{C}}\right)=196 \pm 17 \AA$ and the result of the high temperature $\xi$ fit is $38 \pm 7 \AA$. The correlation lengths are $\xi=1 / \sigma$ with no correction for instrument resolution and are therefore lower bounds. Error bars are standard deviations determined from least squares fitting.

CDW for three dopings at various temperatures. At $T \approx T_{\mathcal{c}}$, the samples with dopings $p=0.23$ and $p=0.25$ exhibit peaks centred at $\delta=0.309 \pm 0.003$ and $0.310 \pm 0.003$ r.l.u. respectively, while no peak is observed for the $p=0.28$ sample. We measured a detailed $T$-dependence of the CDW peak in the $p=0.25\left(T_{c}=45 \mathrm{~K}\right)$ sample (Fig. 3c) to determine the variation of the CDW peak intensity $I_{\mathrm{CDW}}$ and correlation length $\xi$ (see Fig. 4), which we define as the reciprocal of the $\sigma$ parameter of the Gaussian fitted to the CDW peak in $H$. The CDW peak intensity and $\xi$ increase below $T=160 \mathrm{~K}$, however, there is evidence that they remain finite with $\xi=38 \pm 7 \AA$ to the highest temperature investigated $T=200 \mathrm{~K}$. Thus Tl2201 seems to have precursor CDW order as seen in other cuprates such as $\mathrm{LSCO}^{10,36}$ and $\mathrm{YBCO}^{37}$. As the temperature is lowered below $T=160 \mathrm{~K}$ the correlation length increases until $T_{c}$ is reached, at which point it decreases, suggesting that the superconductivity and the CDW interact. Similar behaviour is observed in other cuprates ${ }^{6,36}$. For the $p=0.23$ sample, only two temperatures were measured, $T=$ $T_{c}=56$ and $160 \mathrm{~K}$. In Fig. $3 \mathrm{~b}$, we see that the $H=-0.31$ peak has disappeared at $160 \mathrm{~K}$ peak placing an upper bound $T_{\mathrm{CDW}}$ of $160 \mathrm{~K}$ for this composition. While the small peak away from $H=-0.31$ is most likely spurious scatting, it could also be caused by a temperature dependent $\mathrm{CDW}$ incommensurability, as seen in $\mathrm{LBCO}^{38}$. More investigation would be required to confirm this. For $p=0.28$ (Fig. 3c), no peak is seen even at $T_{c}=22 \mathrm{~K}$ where the signal is expected to be maximal. From our measurements, we deduce the phase diagram shown in Fig. $5 c$, where the black circles represent $T_{\mathrm{CDW}}(p)$, (for $p=0.23$ it is an upper bound) and the critical doping of the $\mathrm{CDW}$ is $p_{\mathrm{CDW}}=0.265 \pm 0.015$, corresponding to $T_{c}=35 \pm 13 \mathrm{~K}$.

\section{Discussion}

Charge density wave order has been observed in many underdoped $(p<0.16)$ cuprates $^{5-11}$, where the ordering wavevectors have been measured as $0.23 \lesssim \delta \lesssim 0.33$. CDW order has recently been observed by $\mathrm{x}$-ray diffraction in two overdoped materials. In $(\mathrm{Bi}, \mathrm{Pb})_{2.12} \mathrm{Sr}_{1.88} \mathrm{CuO}_{6+\delta}(\mathrm{Bi} 2201)$, re-entrant charge order, which is disconnected from the CDW below critical doping is observed $^{29}$ and a CDW is observed in overdoped $\mathrm{La}_{1.79} \mathrm{Sr}_{0.21} \mathrm{CuO}_{4}{ }^{36}$. The behaviour of the peak observed in overdoped $\mathrm{Bi} 2201^{29}$ differs from Tl2201 in that it remains strong up to $250 \mathrm{~K}$ and it is unaffected by the superconductivity. In contrast, the effect of varying temperature on the peak observed in

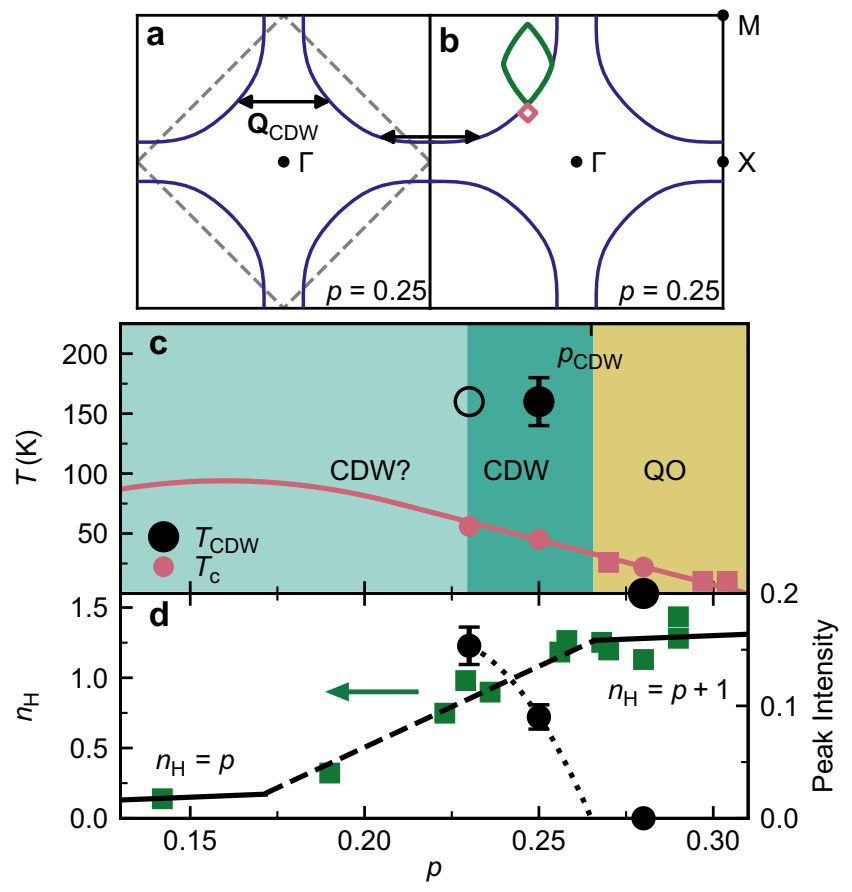

Fig. 5 Charge density wave and Fermi surface reconstruction in

$\mathbf{T l}_{\mathbf{2}} \mathbf{B a}_{\mathbf{2}} \mathbf{C} \mathbf{C} \mathbf{O}_{\mathbf{6}+\boldsymbol{\delta}}$. $\mathbf{a}$ Fermi surface of TI2201 (see Methods), with symmetry labels and volume corresponding to 0.25 doped holes (volume is $1+p$ ). The dashed line is the antiferromagnetic Brillouin zone of the cuprates. CDW order (arrows) may cause a reconstruction yielding electron (red) and hole (green) pockets shown in $\mathbf{b}$. c Phase diagram of overdoped TI2201. CDW order is observed for doping $p<p_{\mathrm{CDW}} \approx 0.265$ (dark green region). High-frequency quantum oscillations are observed for $p \geq 0.2724$ (sand coloured region and red squares) ${ }^{24}$. CDW existence is undetermined in light green region. CDW onset temperatures $T_{C D W}$ are shown by black circles, for $p=0.23$ the open circle represents an upper bound. Red line is superconducting $T_{\mathrm{c}}$ with samples measured here denoted by red filled circles. $\mathbf{d}$ The high-field/low-temperature Hall number 21,51 (green squares). Lines of $n_{H}=p$ and $n_{H}=p+1$ are marked, with connecting dashed line. CDW peak intensity for $T=T_{c}$ (black circles). Dotted line is a guide to the eye passing through black circles and $p_{\mathrm{CDW}}$.

overdoped LSCO $(p=0.21)^{36}$ is qualitatively similar to Tl2201 in that its height drops considerably above $T_{c}$, leaving a small broad component at higher temperatures, and the peak height is reduced on entering the superconducting state. The wavevectors of the CDWs in overdoped Tl2201 $(\delta \approx 0.31)$ and LSCO $(\delta \approx 0.24)$ are similar to those found in underdoped cuprates $(0.23<\delta<0.33)$. In the case of LSCO, $\delta$ does not change much with doping ${ }^{36}$ and $\delta$ for Tl2201 $(0.31)$ is similar to the values found for underdoped $\mathrm{YBCO}^{5,6}$ where $0.31<\delta<0.33$. In this experiment we probe the CDW at $L=2.5$. Unfortunately, scans at different $L$ values were not possible due to experimental constraints (see Supplementary Note 1), thus the detailed $L$-dependence is unknown. We note, however, that in $\mathrm{YBCO}^{6}$ and $\mathrm{LSCO}^{10}$ the CDW is known to be peaked at $L$ equal to halfinteger positions such as $(\delta, 0, n+1 / 2)$. Likely mechanisms causing the corresponding $c$-axis correlations are the interaction of the CDW with pinning $\operatorname{sites}^{39}$ caused by dopant atoms/ quenched disorder or the interactions between the CDW in neighbouring planes ${ }^{40}$.

The CDW order in Tl2201 shows a long correlation length for cuprates (in the absence of 3D order induced by magnetic field or strain) of $\xi=196 \pm 17 \AA$ for $p=0.25$ at $T=T_{c}=45 \mathrm{~K}$. In LSCO $(x=0.21)^{36}$ and YBCO $(p=0.12)^{6}$ the maximum correlation lengths are about 80 and $95 \AA$ respectively. The correlation length 
is likely limited by quenched disorder induced pinning which may be low in Tl2201, as evidenced by its long electronic meanfree-path ${ }^{24}$. We can compare the $H$-integrated area of the CDW Bragg peaks normalised by the $d d$ excitations (see Fig. 1) to other cuprates. We find (see Supplementary Note 4, Supplementary Table 2, and Supplementary Fig. 5) that the $H$-integrated area for $p=0.23 \mathrm{Tl} 2201$ is comparable to $p=0.12 \mathrm{LSCO}$ at $T \approx T_{c}$.

Figure $5 \mathrm{a}, \mathrm{b}$ shows the Fermi surface of Tl2201 $(p=0.25)$ determined from the tight-binding fit of Platé et al. ${ }^{27}$ based on ARPES, with the chemical potential shifted to give a FS area corresponding to $1+p=1.25$. We place $\mathbf{Q}_{\mathrm{CDW}}$ on the same figure at positions where it connects FS states. Johannes and Mazin $^{41}$ have emphasised that Fermi surface nesting rarely determines CDW order other than in quasi-1D systems. Hence it is not surprising that our $\mathbf{Q}_{\mathrm{CDW}}$ does not connect the nested states across the $X$ position. We note, however, that it does approximately connect Fermi surface states along $\Gamma-M$, which have no $k_{z}$ dispersion $^{25}$ and therefore are nested along $k_{z}$. However, this condition does not appear to predict $\mathbf{Q}_{\mathrm{CDW}}$ in other cuprates. Our result is also inconsistent with models (e.g. ref. ${ }^{8,42}$ ) that connect $\mathbf{Q}_{\mathrm{CDW}}$ with hotspot positions, where the FS intersects the magnetic zone boundary (dashed line in Fig. 5a). Thus it appears that determining $\mathbf{Q}_{\mathrm{CDw}}$ requires a strong coupling theory of the electron interactions ${ }^{43}$.

The appearance of the CDW below $p=0.28$ and its increase in amplitude as the doping is lowered (see Fig. 5d) correlates with marked changes in the electronic properties as a function of $p$, in particular $n_{H}^{\infty}$, as shown in Fig. 5d. Within Boltzmann transport theory, $n_{H}^{\infty}$ is simply related to the effective number of carriers and so a reduction in $n_{H}^{\infty}$ could be explained by a reconstruction of the Fermi surface, such as that produced by a CDW.

If the CDW were sufficiently coherent we would expect a reconstruction of the Fermi surface. We have modelled this by adding potentials due to the CDW to a tight-binding model of Tl2201 (see Methods). The result is shown in Fig. 6a. Here, for simplicity, we have assumed a commensurate bi-axial order with $\left|\mathbf{Q}_{\mathrm{CDW}}\right|=1 / 3$ (compared to $\left|\mathbf{Q}_{\mathrm{CDW}}\right| \approx 0.31$ in our experiment) and a very small gap $(2 \mathrm{meV})$. To first approximation, the calculation translates the FS by multiples of $\mathbf{Q}_{\mathrm{CDW}}$ along the $a$ and $b$ axes, with gaps appearing where sections of the original FS are connected by $\mathbf{Q}_{\mathrm{CDW}}$. In this commensurate case, no gaps appear at the other places where the Fermi surfaces cross and so electrons will follow the trajectory of the original FS. The reconstructed FS therefore consists of electron and hole pockets along with open sections of FS as outlined by the coloured lines in Fig. 6a.

Although these pockets have a much lower volume than the original unreconstructed Fermi surface, in the limit that the CDW gap is small, calculations (see Supplementary Note 5 and Supplementary Fig. 6) show that $n_{H}$ will not be strongly affected. However, as the gap grows the electron pocket will shrink and eventually disappear and $n_{H}$ will be reduced to values $\sim p$. The behaviour is similar to the model of Storey ${ }^{17}$ for an antiferromagnetic (pseudogap) reconstruction. Hence, the evolution of $n_{H}^{\infty}$ with $p$ in Tl2201 (Fig. 5d) might be explained by a growth in the CDW gap as $p$ is reduced. This is supported by the observed growth of the $\mathrm{x}$-ray intensity (Fig. 5d) but further $\mathrm{x}$-ray data, at lower $p$, is required to test this proposition.

In YBCO, the occurrence of the CDW with $p$ is accompanied by a change in sign of $n_{H}{ }^{15}$, whereas for Tl2201 $n_{H}$ remains positive for all $p^{21}$. In Fig. $6 \mathrm{~b}$ we show a similar calculation where the Fermi-level has been adjusted to give $p=0.12$. It can be seen that the $p=0.25$ case has larger hole pockets and smaller electron pockets compared to the $p=0.12$ case. In underdoped $(p=0.12)$ YBCO, the hole pocket volume is further reduced by the emergence of the pseudogap ${ }^{13}$. The change in sign of $n_{H}$ in YBCO is
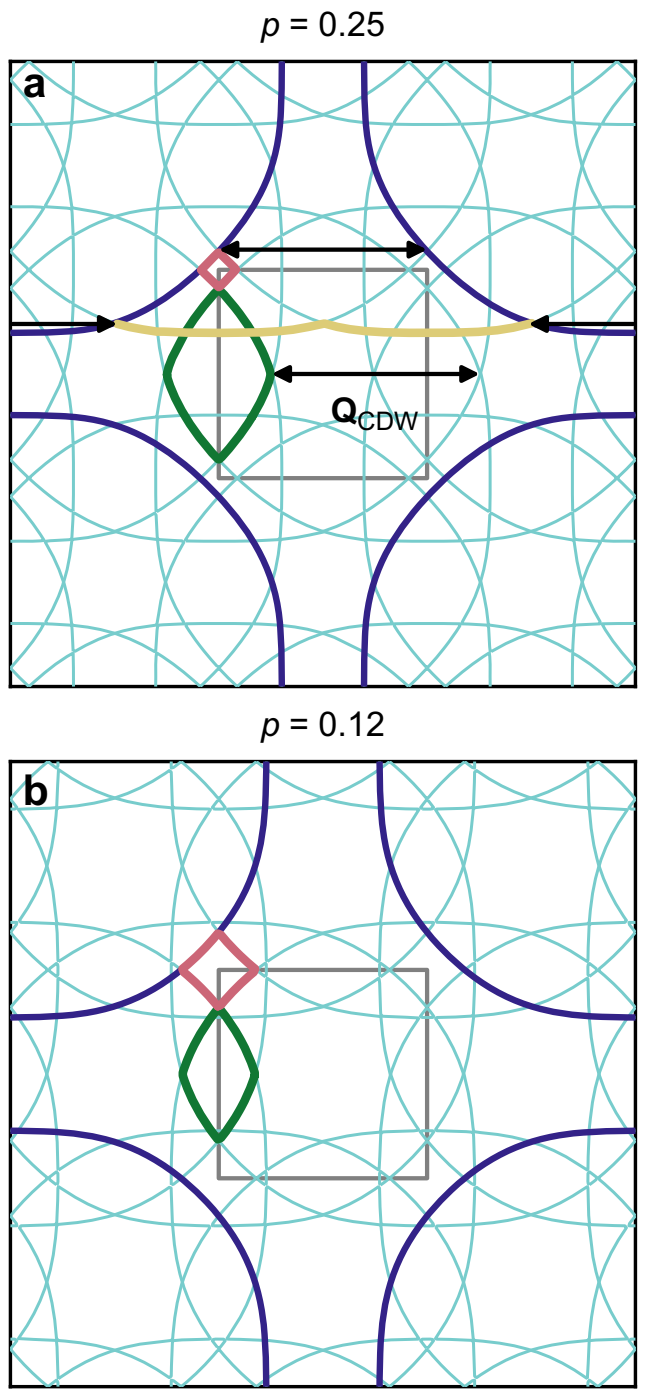

Fig. 6 Model of Fermi surface reconstruction in TI2201. A commensurate $Q=2 \pi / 3$ has been used. $\mathbf{a}$ Shows the reconstruction for $p=0.25$ and $\mathbf{b}$ for $p=0.12$. The original unreconstructed FS is shown in dark blue. Arrows connect positions that are gapped in the reconstructed FS. The reconstructed electron pockets are coloured red, and the hole pockets green. The open FS sections are coloured sand for $p=0.25$. The reduced Brillouin zone (BZ) appropriate to the commensurate order is shown by the small grey rectangle in the centre. The thin turquoise lines show all the contours of the reconstructed FS. For the $p=0.25$ simulation, the hole pockets ( 2 per BZ) contain 0.054 holes and the electron pockets (1 per BZ) 0.003 electrons.

explained by the electron pocket contribution being larger than the hole pocket (see Methods). For T12201, calculations (see Supplementary Note 5 and Supplementary Fig. 6) show that the larger size of the hole pockets and the absence of a pseudogap mean that $n_{H}$ remains positive even when the CDW gap is large.

The large unreconstructed Fermi sheet centred on the $M$ point in Fig. 5b results in quantum oscillations with frequency $F \approx 18 \mathrm{kT}$ which have been observed in Tl2201 for $p \geq 0.27^{22,24}$. The observation of QO is dependent on electrons completing coherent orbits around the Fermi surface. Thus the disappearance of the $18 \mathrm{kT}$ frequency for $p<0.27$ (see Fig. $5 \mathrm{c}$ ) is consistent the CDW causing additional scattering of the orbiting electrons at specific $\mathbf{k}$ vectors. Note that no obvious concomitant increase in the resistivity at $p_{\mathrm{CDW}}$ is observed ${ }^{21}$, so the additional scattering must be quite localised in $\mathbf{k}$. 
The non-observation of lower-frequency QO for $p<0.27^{23,24}$ in Tl2201 suggests that the CDW coherence is not sufficient for electrons to complete cyclotron orbits around the smaller reconstructed pockets with the available magnetic field. This is a much stronger criterion than that required to see reconstruction in the Hall effect ${ }^{44}$.

Spin and charge order or low-frequency fluctuations persist into the overdoped region of the cuprate phase diagram. Lowenergy incommensurate spin fluctuation ${ }^{45}$ and charge density wave order ${ }^{36}$ are seen in overdoped $\mathrm{La}_{2-x} \mathrm{Sr}_{x} \mathrm{CuO}_{4}(p \approx 0.21)$. It is likely that the spin fluctuations are also present in overdoped Tl2201 although they are difficult to measure. Here we show that charge density wave order with a long correlation length is present in Tl2201 at higher dopings disappearing at doping $p_{\mathrm{CDW}}=0.265 \pm 0.015$, corresponding to $T_{c}=35 \pm 13 \mathrm{~K}$. The $\mathrm{CDW}$ appears in a part of the phase diagram where there is no pseudogap, hence showing the two phenomena are not linked, at least in Tl2201. As in other cuprates ${ }^{6}$, superconductivity in Tl2201 causes the CDW correlation length to shorten and hence the CDW is affected by the superconductivity.

Overdoped Tl2201 has a number of interesting quasiparticle properties including an increase in strength of a linear-in- $T$ component to the resistivity as doping is reduced from the edge of the superconducting dome $\mathrm{e}^{21,46}$, quasiparticle scattering rates which are highly anisotropic ${ }^{26}$, a strong variation of Hall number with doping 21 and a magnetoresistance which is much larger than predicted by Boltzmann theory and is insensitive to impurities and magnetic field direction ${ }^{47}$. We have argued that the CDW order is a potential explanation for the variation of the Hall number with doping. Future work is needed to establish whether the other properties mentioned above can be understood in terms of the spin and charge correlations, and the associated scattering of quasiparticles near the Fermi surface.

\begin{abstract}
Methods
Sample growth and preparation. Single crystals of Tl2201 were grown by a selfflux method, similar to that described in ref. ${ }^{48}$. The three samples measured roughly $300 \times 200 \mu \mathrm{m}$, and were hole doped to values of $p=0.23\left(T_{c}=56 \mathrm{~K}\right), 0.25$ $\left(T_{c}=47 \mathrm{~K}\right)$ and $0.28\left(T_{c}=22 \mathrm{~K}\right)$, or samples A, D and G respectively. Sample G was annealed in pure oxygen at $325^{\circ} \mathrm{C}$. Samples A and D were annealed in $0.1 \%$ oxygen in argon at 550 and $500^{\circ} \mathrm{C}$ respectively. Samples were annealled for up to $64 \mathrm{~h}$ and quenched by quickly removing them from the furnace and placing on a copper block. $T_{\mathrm{c}}$ was determined from the mid-point of a.c. susceptibility superconducting transition. Doping values were determined from $T_{c}$ using the relation given in ref. ${ }^{21}$. Tl2201 is tetragonal, with approximate lattice parameters $a=b=3.85 \AA$ and $c=23.1 \AA$ A. The samples had mirror-like surfaces as grown and were not cleaved.
\end{abstract}

Resonant inelastic x-ray scattering. RIXS was carried out at the ID32 beamline at the ESRF, Grenoble, France. Using $x$-ray absorption spectroscopy (XAS), the incident $\mathrm{x}$-ray energy was tuned to the $\mathrm{Cu}-L_{3}$ edge at the absorption peak at $931.6 \mathrm{eV}$. Data were collected with photons polarised linearly, vertical to the scattering plane (LV), in the low resolution/high throughput configuration of the instrument. The energy resolution was further relaxed from 42 to $48 \mathrm{meV}$ by opening the exit slit in order to observe weak elastic scattering. The UB matrix (sample orientation) was determined on ID32 using diffraction from the (002) and (103) Bragg reflections. Scans in the $(H, 0, L)$ plane were made changing sample rotation $\theta$ and detector position $2 \theta$ in the horizontal plane. Except for those shown in Fig. 3a, data were normalised by the orbital excitations, obtained by integrating RIXS spectra in the energy range $1000<E<3000 \mathrm{meV}$, resulting in a dimensionless ratio of intensities. Error bars on RIXS intensity and the elastic intensity were obtained by taking advantage of single photon counting at ID32, assuming Poisson statistics. Error bars in the fitted parameters were obtained from least square fitting and represent one standard deviation.

The low-energy excitations of the RIXS spectra, where $0<E<300 \mathrm{meV}$ were fitted to three features. The elastic peaks were fitted to a Gaussian with a FWHM fixed to the the instrument resolution. This was used to determine zero energy. An inelastic Gaussian peak with FWHM fixed to instrument resolution was fitted to the $\mathrm{CuO}$ stretching phonon where present (discussed more in main text). The magnetic paramagnon excitation was fitted to a damped harmonic oscillator that was numerically convoluted with a Gaussian with FWHM set to instrument resolution. To account any higher energy excitations, a linear background was used.
Fermi surface reconstruction. The simulations of Fermi surface reconstruction in Fig. 6 was based on the ARPES derived tight-binding parameterisation of the FS of Tl220127; $\varepsilon(K)=\mu+\frac{t_{1}}{2}\left(\cos k_{x}+\cos k_{y}\right)+t_{2} \cos k_{x} \cos k_{y}+\frac{t_{3}}{2}\left(\cos 2 k_{x}+\right.$ $\left.\cos 2 k_{y}\right)+\frac{t_{4}}{2}\left(\cos 2 k_{x} \cos k_{y}+\cos k_{x} \cos 2 k_{y}\right)+t_{5} \cos 2 k_{x} \cos 2 k_{y}$, with $t_{1}=-0.725, t_{2}=0.302, t_{3}=0.0159, t_{4}=-0.0805$ and $t_{5}=0.0034$; for $p=0.25 \mu=0.2382$ and $p=0.12 \mu=0.1998$. All energies are expressed in $\mathrm{eV}$ with lattice parameter $a=1$. Small CDW potentials with $V_{0}=0.002, \mathbf{Q}_{\mathrm{CDW}}=$ $(2 \pi / 3,0)$ and $\mathbf{Q}_{\mathrm{CDW}}=(0,2 \pi / 3)$ were introduced using the method described in ref. ${ }^{49}$, but with a $\mathbf{k}$-independent potential. This translates copies of the FS by multiples of $\mathbf{Q}_{\mathrm{CDW}}$ in the $\mathbf{a}$ and $\mathbf{b}$ directions and introduces small gaps on those parts of the FS connected by $\mathbf{Q}_{\mathrm{CDW}}$. In the incommensurate case, which corresponds to our experimental $\left|\mathbf{Q}_{\mathrm{CDW}}\right| \approx 0.31$ r.l.u., small gaps will also be present where the other sections of FS cross. However, these gaps will generally be small so the electrons will be able to tunnel across in moderate fields and hence the transport will be largely unaffected ${ }^{49}$.

In the low-field limit, $n_{H}=(B / e) \sigma_{x x}^{2} / \sigma_{x y}$, where $\sigma_{x x}$ and $\sigma_{x y}$ are the longitudinal and Hall conductivities respectively and $B$ is the magnetic field. The sign of $n_{H}$ is therefore the same as $\sigma_{x y}$, and which is the sum of positive and negative contributions from the hole and electron pockets respectively which in turn depend predominantly on the mean-free-path on each pocket. The open sections of the Fermi surface will have only a small contribution to $\sigma_{x y}$ as their contribution is zero if the mean-free-path is isotropic ${ }^{50}$. These open sections will however, make a substantial contribution to $\sigma_{x x}$ so will affect the magnitude of $n_{H}$ (see Supplementary Note 5 for details of the $n_{H}$ calculations).

\section{Data availability}

All relevant data are available from the corresponding authors upon reasonable request and at https://doi.org/10.15151/ESRF-DC-646880761

Received: 27 August 2021; Accepted: 10 January 2022; Published online: 28 January 2022

\section{References}

1. Keimer, B., Kivelson, S. A., Norman, M. R., Uchida, S. \& Zaanen, J. From quantum matter to high-temperature superconductivity in copper oxides. Nature 518, 179 (2015).

2. Timusk, T. \& Statt, B. The pseudogap in high-temperature superconductors: an experimental survey. Rep. Prog. Phys 62, 61 (1999).

3. Tallon, J. \& Loram, J. The doping dependence of $T^{\star}$ - What is the real high- $T_{c}$ phase diagram? Physica C Supercond. 349, 53 (2001).

4. Tallon, J. L., Storey, J. G., Cooper, J. R. \& Loram, J. W. Locating the pseudogap closing point in cuprate superconductors: Absence of entrant or reentrant behavior. Phys. Rev. B 101, 174512 (2020).

5. Ghiringhelli, G. et al. Long-range incommensurate charge fluctuations in (Y,Nd) $\mathrm{Ba}_{2} \mathrm{Cu}_{3} \mathrm{O}_{6+x}$. Science 337, 821 (2012).

6. Chang, J. et al. Direct observation of competition between superconductivity and charge density wave order in $\mathrm{YBa}_{2} \mathrm{Cu}_{3} \mathrm{O}_{6.67}$. Nat. Phys. 8, 871 (2012).

7. da Silva Neto, E. H. et al. Ubiquitous interplay between charge ordering and high-temperature superconductivity in cuprates. Science 343, 393 (2013)

8. Comin, R. et al. Charge order driven by Fermi-arc instability in $\mathrm{Bi}_{2} \mathrm{Sr}_{2-x} \mathrm{La}_{x} \mathrm{CuO}_{6+\delta}$. Science 343, 390 (2013).

9. Tabis, W. et al. Charge order and its connection with Fermi-liquid charge transport in a pristine high- $T_{c}$ cuprate. Nat. Commun. 5, 5875 (2014).

10. Croft, T. P., Lester, C., Senn, M. S., Bombardi, A. \& Hayden, S. M. Charge density wave fluctuations in $\mathrm{La}_{2-x} \mathrm{Sr}_{x} \mathrm{CuO}_{4}$ and their competition with superconductivity. Phys. Rev. B 89, 224513 (2014).

11. Thampy, V. et al. Rotated stripe order and its competition with superconductivity in $\mathrm{La}_{1.88} \mathrm{Sr}_{0.12} \mathrm{CuO}_{4}$. Phys. Rev. B 90, 100510 (2014).

12. Harrison, N. \& Sebastian, S. E. Protected nodal electron pocket from multiple$\mathbf{Q}$ ordering in underdoped high temperature superconductors. Phys. Rev. Lett. 106, 226402 (2011).

13. Allais, A., Chowdhury, D. \& Sachdev, S. Connecting high-field quantum oscillations to zero-field electron spectral functions in the underdoped cuprates. Nat. Commun. 5, 5771 (2014).

14. LeBoeuf, D. et al. Electron pockets in the Fermi surface of hole-doped high- $T_{c}$ superconductors. Nature 450, 533 (2007).

15. LeBoeuf, D. et al. Lifshitz critical point in the cuprate superconductor $\mathrm{YBa}_{2} \mathrm{Cu}_{3} \mathrm{O}_{y}$ from high-field Hall effect measurements. Phys. Rev. B 83, 054506 (2011).

16. Badoux, S. et al. Change of carrier density at the pseudogap critical point of a cuprate superconductor. Nature 531, 210 (2016).

17. Storey, J. G. Hall effect and Fermi surface reconstruction via electron pockets in the high- $T_{c}$ cuprates. EPL 113, 27003 (2016). 
18. Wade, J. M., Loram, J. W., Mirza, K. A., Cooper, J. R. \& Tallon, J. L. Electronic specific heat of $\mathrm{Tl}_{2} \mathrm{Ba}_{2} \mathrm{CuO}_{6+\delta}$ from $2 \mathrm{~K}$ to $300 \mathrm{~K}$ for $0 \leq \delta \leq 0.1$. J. Supercond. 7 , 261 (1994).

19. Fujiwara, K. et al. ${ }^{63} \mathrm{Cu}$ knight shift study in high- $T_{c}$ superconductor $\mathrm{Tl}_{2} \mathrm{Ba}_{2} \mathrm{CuO}_{6+y}$ with a single $\mathrm{CuO}_{2}$ layer. J. Phys. Soc. Jpn. 59, 3459 (1990).

20. Kambe, S., Yasuoka, H., Hayashi, A. \& Ueda, Y. NMR study of the spin dynamics in $\mathrm{Tl}_{2} \mathrm{Ba}_{2} \mathrm{CuO}_{6+y}\left(T_{c}=85 \mathrm{~K}\right)$. Phys. Rev. B 47, 2825 (1993).

21. Putzke, C. et al. Reduced Hall carrier density in the overdoped strange metal regime of cuprate superconductors. Nat. Phys. 17, 826 (2021).

22. Vignolle, B. et al. Quantum oscillations in an overdoped high- $T_{c}$ superconductor. Nature 455, 952 (2008).

23. Bangura, A. F. et al. Fermi surface and electronic homogeneity of the overdoped cuprate superconductor $\mathrm{Tl}_{2} \mathrm{Ba}_{2} \mathrm{CuO}_{6+\delta}$ as revealed by quantum oscillations. Phys. Rev. B 82, 140501 (2010).

24. Rourke, P. M. C. et al. A detailed de Haas-van Alphen effect study of the overdoped cuprate $\mathrm{Tl}_{2} \mathrm{Ba}_{2} \mathrm{CuO}_{6+\delta}$. New J. Phys. 12, 105009 (2010).

25. Hussey, N. E., Abdel-Jawad, M., Carrington, A., Mackenzie, A. P. \& Balicas, L. A coherent three-dimensional Fermi surface in a high-transition-temperature superconductor. Nature 425, 814 (2003).

26. Abdel-Jawad, M. et al. Anisotropic scattering and anomalous normal-state transport in a high-temperature superconductor. Nat. Phys. 2, 821 (2006).

27. Platé, M. et al. Fermi surface and quasiparticle excitations of overdoped $\mathrm{Tl}_{2} \mathrm{Ba}_{2} \mathrm{CuO}_{6+\delta}$. Phys. Rev. Lett. 95, 077001 (2005).

28. Lin, J. et al. Strongly correlated charge density wave in $\mathrm{La}_{2-x} \mathrm{Sr}_{x} \mathrm{CuO}_{4}$ evidenced by doping-dependent phonon anomaly. Phys. Rev. Lett. 124, 207005 (2020)

29. Peng, Y. Y. et al. Re-entrant charge order in overdoped $(\mathrm{Bi}, \mathrm{Pb})_{2.12} \mathrm{Sr}_{1.88} \mathrm{CuO}_{6+\delta}$ outside the pseudogap regime. Nat. Mater. 17, 697 (2018).

30. He, Y. et al. Persistent low-energy phonon broadening near the charge-order $q$ vector in the bilayer cuprate $\mathrm{Bi}_{2} \mathrm{Sr}_{2} \mathrm{CaCu}_{2} \mathrm{O}_{8+\delta}$. Phys. Rev. B 98, 035102 (2018).

31. Le Tacon, M. et al. Dispersive spin excitations in highly overdoped cuprates revealed by resonant inelastic x-ray scattering. Phys. Rev. B 88, 020501 (2013).

32. Devereaux, T. et al. Directly characterizing the relative strength and momentum dependence of electron-phonon coupling using resonant inelastic x-ray scattering. Phys. Rev. X 6, 041019 (2016).

33. Chaix, L. et al. Dispersive charge density wave excitations in $\mathrm{Bi}_{2} \mathrm{Sr}_{2} \mathrm{CaCu}_{2} \mathrm{O}_{8+\delta}$. Nat. Phys. 13, 952 (2017).

34. Peng, Y. Y. et al. Enhanced electron-phonon coupling for charge-density-wave formation in $\mathrm{La}_{1.8-x} \mathrm{Eu}_{0.2} \mathrm{Sr}_{x} \mathrm{CuO}_{4+\delta}$. Phys. Rev. Lett. 125, 097002 (2020).

35. Boschini, F. et al. Dynamic electron correlations with charge order wavelength along all directions in the copper oxide plane. Nat. Commun. 12, 597 (2021).

36. Miao, H. et al. Charge density waves in cuprate superconductors beyond the critical doping. npj Quant. Mater. 6, 31 (2021).

37. Arpaia, R. et al. Dynamical charge density fluctuations pervading the phase diagram of a Cu-based high- $T_{c}$ superconductor. Science 365, 906 (2019).

38. Miao, $\mathrm{H}$. et al. High-temperature charge density wave correlations in $\mathrm{La}_{1.875} \mathrm{Ba}_{0.125} \mathrm{CuO}_{4}$ without spin-charge locking. Proc. Natl Acad. Sci. USA. 114, 12430 (2017).

39. Caplan, Y. \& Orgad, D. Dimensional crossover of charge-density wave correlations in the cuprates. Phys. Rev. Lett. 119, 107002 (2017).

40. Hücker, M. Structural aspects of materials with static stripe order. Physica C Supercond. 481, 3 (2012).

41. Johannes, M. D. \& Mazin, I. I. Fermi surface nesting and the origin of charge density waves in metals. Phys. Rev. B 77, 165135 (2008).

42. Pépin, C., de Carvalho, V. S., Kloss, T. \& Montiel, X. Pseudogap, charge order, and pairing density wave at the hot spots in cuprate superconductors. Phys. Rev. B 90, 195207 (2014).

43. Kivelson, S. A. et al. How to detect fluctuating stripes in the high-temperature superconductors. Rev. Mod. Phys. 75, 1201 (2003).

44. Gannot, Y., Ramshaw, B. J. \& Kivelson, S. A. Fermi surface reconstruction by a charge density wave with finite correlation length. Phys. Rev. B 100, 045128 (2019).

45. Lipscombe, O. J., Hayden, S. M., Vignolle, B., McMorrow, D. F. \& Perring, T. G. Persistence of high-frequency spin fluctuations in overdoped superconducting $\mathrm{La}_{2-x} \mathrm{Sr}_{x} \mathrm{CuO}_{4}(x=0.22)$. Phys. Rev. Lett. 99, 067002 (2007).
46. Hussey, N. E. Phenomenology of the normal state in-plane transport properties of high- $T_{c}$ cuprates. J. Phys. Condens. Matter 20, 123201 (2008).

47. Ayres, J. et al. Incoherent transport across the strange-metal regime of overdoped cuprates. Nature 595, 661 (2021).

48. Tyler, A. W. An Investigation into the Magnetotransport Properties of Layered Superconducting Perovskites Ph.D. thesis, Cambridge Univ. (1998).

49. Harrison, N. Near doping-independent pocket area from an antinodal Fermi surface instability in underdoped high temperature superconductors. Phys. Rev. Lett. 107, 186408 (2011).

50. Ong, N. P. Geometric interpretation of the weak-field Hall conductivity in two-dimensional metals with arbitrary Fermi surface. Phys. Rev. B 43, 193 (1991).

51. Mackenzie, A. P., Julian, S. R., Sinclair, D. C. \& Lin, C. T. Normal-state magnetotransport in superconducting $\mathrm{Tl}_{2} \mathrm{Ba}_{2} \mathrm{CuO}_{6+\delta}$ to millikelvin temperatures. Phys. Rev. B 53, 5848 (1996).

\section{Acknowledgements}

We acknowledge useful discussions with Nigel Hussey, Steve Kivelson and Cyril Proust Liam Malone contributed to the sample growth. AC and SMH acknowledge funding from the UK Engineering and Physical Sciences Research Council under grant EP/ R011141/1.

\section{Author contributions}

The RIXS experiment was conceived by SMH. Samples were grown and characterised by JA, AC and JRC. RIXS experiments were performed by CCT, MZ, KK, FY-H and SMH. The RIXS data was analysed by CCT and SMH. Fermi surface reconstruction calculation was performed by AC. The manuscript was written by CCT, AC and SMH with input from all authors.

\section{Competing interests}

The authors declare no competing interests.

\section{Additional information}

Supplementary information The online version contains supplementary material available at https://doi.org/10.1038/s41467-022-28124-y.

Correspondence and requests for materials should be addressed to A. Carrington or S. M. Hayden.

Peer review information Nature Communications thanks the anonymous, reviewer(s) for their contribution to the peer review of this work. Peer reviewer reports are available

Reprints and permission information is available at http://www.nature.com/reprints

Publisher's note Springer Nature remains neutral with regard to jurisdictional claims in published maps and institutional affiliations.

Open Access This article is licensed under a Creative Commons Attribution 4.0 International License, which permits use, sharing, adaptation, distribution and reproduction in any medium or format, as long as you give appropriate credit to the original author(s) and the source, provide a link to the Creative Commons license, and indicate if changes were made. The images or other third party material in this article are included in the article's Creative Commons license, unless indicated otherwise in a credit line to the material. If material is not included in the article's Creative Commons license and your intended use is not permitted by statutory regulation or exceeds the permitted use, you will need to obtain permission directly from the copyright holder. To view a copy of this license, visit http://creativecommons.org/ licenses/by/4.0/.

(C) The Author(s) 2022 